\title{
EchoGéo
}

31 | 2015

Glocal Ethiopia

\section{Le bétail des Somali d'Éthiopie : la mondialisation sur les marges}

Géraldine Pinauldt

\section{OpenEdition}

Journals

Édition électronique

URL : https://journals.openedition.org/echogeo/14163

DOI : 10.4000/echogeo.14163

ISSN : 1963-1197

Éditeur

Pôle de recherche pour l'organisation et la diffusion de l'information géographique (CNRS UMR 8586)

Référence électronique

Géraldine Pinauldt, « Le bétail des Somali d'Éthiopie : la mondialisation sur les marges », EchoGéo [En ligne], 31 | 2015, mis en ligne le 10 avril 2015, consulté le 08 décembre 2022. URL : http:// journals.openedition.org/echogeo/14163; DOI : https://doi.org/10.4000/echogeo.14163

Ce document a été généré automatiquement le 31 juillet 2021.

\section{(c) (i) (3)}

Creative Commons - Attribution - Pas d'Utilisation Commerciale - Pas de Modification 4.0 International - CC BY-NC-ND 4.0

https://creativecommons.org/licenses/by-nc-nd/4.0/ 


\title{
Le bétail des Somali d'Éthiopie : la mondialisation sur les marges
}

\author{
Géraldine Pinauldt
}

\section{Introduction}

1 S'étendant sur un quart du territoire éthiopien, l'État Régional Somali d'Éthiopie (ERSE), abrite une population transfrontalière dont le territoire s'étend sur plusieurs autres États voisins de l'Éthiopie (Somaliland, Kenya, Djibouti et Somalie). Longtemps cible consentante de l'irrédentisme somalien, les Somali d'Éthiopie disposent aujourd'hui officiellement, à l'instar des autres communautés ethnolinguistiques éthiopiennes, d'une forme d'autonomie de gestion au sein de l'Éthiopie fédérale. L'ambiguïté de l'allégeance des Somali-éthiopiens est pourtant entretenue au niveau éthiopien comme au niveau somali. Ce rapport compliqué du pouvoir éthiopien aux ressources, à l'espace et à la population de l'ERSE se révèle particulièrement par l'étude de sa principale ressource: le bétail.

2 Le bétail de la région somali d'Éthiopie constitue une part non négligeable ${ }^{1}$ du bétail exporté vers la péninsule arabique depuis les ports somali et ce depuis l'installation des Britanniques à Aden en $1839^{2}$. Ce système commercial régional (qui organise la production et les réseaux d'exportations convergeant vers Berbera au Somaliland) se consolide dans les années 1950 avec l'augmentation des pèlerins à la Mecque, ellemême corrélée à l'envolée des revenus pétroliers de l'Arabie saoudite. Mobile et transfrontalier, le bétail des Somali est exporté via un système souple et réticulaire qui longtemps a totalement échappé au contrôle de l'État éthiopien. Le succès de ce système fondé sur la libre entreprise dans le contexte d'une forte solidarité collective constitue un paradoxe au sein de cet État éthiopien longtemps très centralisé et à l'économie planifiée. Inversement, les investissements éthiopiens dans le secteur animal n'ont jamais dépassé quelques pourcents des dépenses agricoles du pays.

3 L'ERSE peut être considéré comme un État régional périphérique, en raison de la perception réciproque d'une distance culturelle entre Somali-éthiopiens et autres 
Éthiopiens : musulmans, nomades, éleveurs dans les basses terres de l'est ; chrétiens, sédentaires et agriculteurs dans les hautes terres. Cette opposition, si elle essentialise le réel, organise cependant de manière très forte l'espace de l'altérité. Cette distance se traduit politiquement: le parti somali au pouvoir, le SPDP (Somali People Democratic Parti), comme pour d'autres régions périphérique, n'est associé que de manière externe au parti fédéral coalisé l'EPRDF (Ethiopian People's Revolutionay Democratic Front), mais n'en est pas partie intégrante, freinant ainsi les possibilités de lobby pastoral au sein du système fédéral. Pourtant dans le cas des exportations de bétail vivant vers l'Arabie, c'est l'État éthiopien qui est périphérique : contrôlées, elles se placeraient en troisième position des exportations éthiopiennes, contre la $7^{\mathrm{e}}$ place aujourd'hui et la $19^{\mathrm{e}} \mathrm{il}$ y a 15 ans. L'évolution de la part du bétail vivant dans l'export éthiopien témoigne néanmoins de sa progressive et partielle intégration aux recettes nationales.

Les tentatives de contrôle et de punition des exportations informelles de bétail ont eu des résultats mitigés pour l'est éthiopien malgré leur renforcement. C'est l'élaboration de normes pour le commerce international qui va donner à l'échelon national les moyens d'un contrôle étatique, d'abord via les services vétérinaires puis par des tentatives de contrôle du commerce en favorisant l'introduction de nouveaux acteurs privés.

5 La mondialisation normative peut être définie par une phase de la mondialisation qui tend à fluidifier le commerce international par l'introduction de normes universelles. Elle est mise en œuvre parallèlement à la création de l'OMC et a pour particularité de réintroduire des barrières non tarifaires au commerce international. Dans le cas du commerce d'exportation de bétail en Éthiopie, sa mise en place se traduit dans les années 2000 par l'apparition de l'échelle nationale (État fédéral) dans la filière animale d'exportation de l'ERSE. On ne peut parler dans ce cas de renforcement de l'État central qui on l'a vu était absent du secteur; il n'y a pas plus de renforcement du pouvoir administratif local. Plus que des jeux d'échelles administratives de pouvoir, c'est le passage d'une économie informelle à une économie formelle qui est en jeu via l'aménagement par des infrastructures de l'espace commercial, dans une région où la population et les acteurs de l'économie ne se sentent pas éthiopiens, alors que les recettes des exportations légales sont collectées au niveau fédéral. Avec l'apparition de contraintes sanitaires fortes à l'exportation, depuis l'embargo sanitaire imposé par les pays de la péninsule arabique au bétail de la Corne de l'Afrique entre 1998 et 2009, l'État éthiopien va avoir une opportunité d'intervenir dans un secteur qui jusqu'alors lui échappait. Au-delà de la résistance populaire à l'insertion dans l'économie formelle, un autre élément vient modérer les efforts éthiopiens d'intégration de l'économie somali. L'État éthiopien est le plus structuré de la région, pourtant sa situation d'enclavement rend vains les efforts éthiopiens d'alignement sur les normes, puisque ce sont les certificats produits dans les ports d'exportation, par d'autres autorités sanitaires ${ }^{3}$, qui sont reconnus outre-golfe d'Aden, outre Mer rouge. Aussi peut-on se demander dans quelle mesure la tentative éthiopienne de rétablir un contrôle sur le territoire et les ressources de l'ERSE aboutit, et dans quelle mesure il n'existe pas après la crise deux formes de commerce associées à deux formes de perception et d'aménagement du territoire. Ces deux formes définissent deux utilisations de l'espace : l'une encore en structuration par l'État par délégation à des collectivités territoriales dans un mouvement de déconcentration plus que de décentralisation ${ }^{4}$, les organisations internationales et les acteurs choisis par les deux premiers, l'autre, survivance encore puissante du commerce qui s'organise selon des faisceaux 
relationnels réticulaires. Quelle va être la relation, notamment spatiale, entre ces deux formes de commerce? L'alignement sur des normes mondiales du commerce d'exportation de bétail en l'éthiopie se traduit-il par l'intégration de la région somali ou au contraire par son plus grand isolement du monde éthiopien avec le renforcement d'une frontière intérieure?

On verra dans un premier temps comment s'est effectuée la mise en mondialisation de ce secteur pour l'Éthiopie, en montrant le rôle qu'ont joué les organisations internationales normatives ou de développement, et autres agences d'aide. Dans un second temps, on rappellera le caractère opérationnel d'une frontière culturelle entre le monde somali et les hautes terres éthiopiennes, particulièrement pertinente dans le cas du commerce de bétail et que matérialisent les politiques sectorielles éthiopiennes. L'exemple de la filiale animale du groupe MIDROC dans l'est-éthiopien illustrera les rouages de la mise en mondialisation du secteur animal éthiopien et ses conséquences sur le territoire éthiopien.

\section{La "mise en mondialisation" du commerce de bétail en Éthiopie}

\section{Les exportations de bétail : une filière de contrebande selon l'Éthiopie, un commerce mondialisé selon les Somali}

7 En quadruplant ses importations entre 1976 et 1980, l'Arabie Saoudite, premier importateur mondial d'ovins vivants, entre dans un commerce mondialisé jetant ainsi, sans transition, son partenaire traditionnel somalien (dont les exportations comprennent le bétail des Somali-éthiopiens) dans la concurrence internationale. Aux fournisseurs habituels du mouton sacrificiel que sont la Somalie (drainant le bétail de l'ensemble de la péninsule Somali) et dans une moindre mesure le Soudan, la Syrie et la Turquie, s'ajoutent à partir des années 1980 l'Australie, la Nouvelle Zélande et l'Uruguay pour ne citer que les principaux. L'Éthiopie, bien qu'elle abrite l'un des plus gros cheptels du continent, n'a jamais été comptabilisée comme un exportateur important de bétail. On observe en effet en Éthiopie d'une part le marché local, à destination des grandes villes et alimenté par le bétail des hautes terres (ovin ou bovin), et d'autre part le marché international, qui exporte le bétail des régions frontalières, en premier lieu le bétail élevé dans la région somali d'Éthiopie. En raison du caractère transfrontalier des populations qui contrôlent le marché, les exportations échappent au secteur formel et à la comptabilité nationale. Marché domestique et marché d'exportation n'entrent donc pas en concurrence directe jusqu'à une période récente, dans la mesure où à un marché correspondent des zones de production et des cultures commerciales distinctes.

8 Jusqu'à la fin des années 1990, les exportateurs de bétail dans la Corne sont principalement privés et somali. Les politiques et investissements publics éthiopiens n'ont jusqu'alors que peu concerné le secteur animal. De plus, les politiques sectorielles concernent très peu l'Éthiopie orientale, à l'exception du TLDP (Third Livestock Development Project, 1975-1986). Son volet oriental JIRDU (Jijiga Rangeland Development Unit) est destiné à créer une circulation intérieure à l'Éthiopie du bétail afin de le détourner des filières somaliennes. Ce projet est considérablement freiné par la guerre éthio-somalienne de 1977. L'embargo de 1998 donne l'occasion à une série de nouveaux 
acteurs (privés, étatiques et supra-étatiques) de découvrir l'importance des échanges et d'y prendre place. À partir de cette date, les restructurations sont multiples en termes d'échelles et d'acteurs. L'embargo est l'occasion d'une mise aux normes du secteur animal en vue de son ouverture aux marchés internationaux. Aussi cet alignement sur des normes sanitaires et commerciales internationales bénéficie-il potentiellement à chaque acteur si l'on considère qu'il peut permettre de rattraper le retard du secteur. L'État éthiopien capte des financements, et les acteurs privés auparavant coupés du bassin de production le plus important - la région somali d'Éthiopie, voient dans la mise aux normes du marché mondial de viande et d'animaux vivants une occasion de concurrencer les grands négociants somali sur leurs terres.

De ce point de vue, avec son entrée tardive dans la mondialisation, l'État éthiopien présente un retard d'adaptation important par rapport aux filières organisées de l'exportation de bétail, aux acteurs anciens dont les pratiques commerciales sont libérales au point d'être informelles.

\section{8, une année charnière dans la mise en mondialisation normative du secteur animal}

$10 \mathrm{Au}$ cours de l'année 1998 on observe une conjonction d'événements à l'échelle mondiale, dans la péninsule arabique et en Éthiopie, qui favorisent la mise en mondialisation de l'Éthiopie et placent l'État dans un rôle décisionnel et actif nouveau dans le secteur animal. En effet, de nouvelles normes internationales se mettent en place qui modifient les règles sanitaires et commerciales des exportations. Leurs conséquences trouvent une traduction à l'échelle de l'espace commercial régional (Corne de l'Afrique/Moyen-Orient), et aux échelles nationales. Ces évolutions, pour l'Éthiopie, sont rythmées par la temporalité de la construction politique et économique de l'espace éthiopien post-Derg et plus particulièrement par la libéralisation progressive de l'économie.

\section{Accord sanitaire et phytosanitaire (Accord SPS) OIE-OMC de 1998}

11 Dans le domaine de la santé animale, l'Organisation internationale de la santé animale (OIE) est le producteur de normes. Ces normes prennent une consistance nouvelle avec la signature, en 1998, d'un premier accord entre l'OIE et l'Organisation mondiale du commerce (OMC). L'Accord SPS de l'OMC établit le nouveau rôle de l'OIE qui doit, à des fins d'harmonisation, produire normes, directives et recommandations internationales sur la base desquelles les membres de l'OMC établiront leurs mesures sanitaires. En 2004, un accord de coopération entre l'OIE et la FAO s'appuie sur l'accord SPS de 1998. Cette nouvelle coopération des organisations spécifie le rôle de l'OIE comme producteur de normes et celui de la FAO comme maître d'œuvre de ces normes par l'intermédiaire de ses programmes.

\section{Processus d'intégration de l'Arabie saoudite à l'OMC (1995-2005) et réorganisation des importations pour le Hajj de 1998}

En 1995, l'Arabie saoudite entame sa procédure d'adhésion à l'OMC dont elle devient membre en 2005. Son processus d'intégration à l'OMC induit des réformes visant à aligner le fonctionnement de son commerce sur les standards internationaux. Dans ce 
cadre, les importations de bétail sacrificiel pour le Hajj sont confiées en 1998 à la Banque islamique de développement (BID), chargée de sélectionner un nombre restreint d'importateurs sur un appel d'offres annuel. Ces importations étaient jusqu'alors le fait d'un grand nombre d'entrepreneurs aux techniques commerciales moins encadrées. Ce bouleversement se répercute sur l'ensemble de la filière commerciale, notamment dans la Corne de l'Afrique dépendante de ce marché. Cette réorganisation en aval est l'occasion pour des investisseurs saoudiens de pénétrer plus en amont le territoire de production en coupant la chaîne de négociants, intermédiaires et agents somali afin de tenter de contrôler leur approvisionnement et de proposer ainsi un tarif plus compétitif lors de l'appel d'offre de la BID.

\section{Corne de l'Afrique : embargo sanitaire en 1998}

Les exportations vers l'Arabie saoudite du bétail élevé dans les régions somali connaissent une envolée avec la réouverture du port de Berbera et la stabilisation du Somaliland et de la région somali d'éthiopie au début des années 1990. C'est dans un libéralisme total que s'organise la filière à cette époque : le marché d'animaux vivants en région somali d'Éthiopie est libéral de fait dans la mesure où l'économie pastorale échappe au contrôle étatique. Au Somaliland, la liberté d'entreprendre est au centre de la construction de ce petit État et sera inscrite en 1997 au préambule de son projet de Constitution. Un embargo sanitaire imposé par l'Arabie Saoudite arrête brutalement cette envolée économique en 1998. Il est suivi d'un deuxième embargo de l'ensemble des États de la péninsule arabique en 2000 et qui dure jusqu'en 2009. Ces embargos et l'obligation de certification sanitaire des animaux exportés imposés par les pays du Golfe permettent à l'Éthiopie de capter des financements internationaux importants afin de structurer son secteur animal à la fois en termes vétérinaires et commerciaux. La normalisation du secteur provoque une crise diplomatico-économique entre le Somaliland et Djibouti qui se disputent l'ouverture d'une station de quarantaine d'exportation de portée régionale reconnue par l'Arabie Saoudite (Pinauldt, 2009).

\section{Éthiopie : création de la Livestock Marketing Authority en 1998}

En Éthiopie, le nouveau régime prend conscience de la singularité des régions pastorales et semble envisager une très progessive intégration du secteur animal de ces espaces. En 1998, la Livestock Marketing Authority (LMA) est créée sous l'égide du Ministère du Commerce afin de promouvoir les exportations. Dans l'Ethiopian Herald cette année-là, des articles très réguliers insistent sur le fait que le bétail est une ressource encore non exploitée, un potentiel important, que les éleveurs et toute la filière n'ont aucune conscience des exigences des importateurs et que la région somali d'Éthiopie est un espace vierge ${ }^{5}$. Parallèlement, le service vétérinaire du Ministère de l'Agriculture et du développement rural (MoArd) est mandaté pour contrôler les maladies animales transfrontalières (Transboundary animal diseases - TAD) et délivrer les certificats vétérinaires nécessaires lors de l'exportation. Pour cela, un certain nombre de stations de quarantaine animale seront planifiées, dont seule celle de Dire Dawa fonctionne à partir de 2004, sur des financements USAID. Le bétail de la région somali constitue la ressource locale la plus importante pour la population. C'est pourtant l'administration fédérale qui plannifie les infrastructures d'exportations et collecte les taxes, ce qui contribue à accroître la défiance populaire envers l'État 
éthiopien et n'encourage pas les exportateurs à intégrer leur commerce à l'économie formelle.

15 S'il est manifeste que les tentatives de captation du commerce de bétail par l'État éthiopien évoluent au rythme des étapes de la stabilisation du nouveau régime depuis les années 1990, c'est en 1998 que convergent les éléments permettant la mise en mondialisation de ce secteur en Éthiopie. L'embargo saoudien donne une occasion unique au régime d'intervenir dans les filières: l'apparition de contraintes sanitaires fortes à l'exportation bénéficie tout naturellement à l'Éthiopie. Malgré le handicap de l'enclavement, elle est naturellement en meilleure position que ses voisins, avec l'administration la plus structurée de la région, la réception d'une aide importante dans le développement de services vétérinaires depuis les années 1980 (Faye, 1990), une position de leader politique régional, et la population humaine et animale la plus importante de cet ensemble.

\section{La rencontre des investissements sectoriels américains et des intérêts éthiopiens}

Les principaux agents de cette phase normative de la mondialisation sont ceux qui mettent en œuvre les nouvelles contraintes réglementaires - au risque de paraître paradoxal - et assistent les États dans leur application. On peut isoler un triptyque planificateur: organisation internationale et/ou État/bailleur; gouvernement occidental et/ou organisation internationale/opérateur; ONG et/ou entreprise privée. Les modalités d'articulation des trois parties et la part de pouvoir de l'une ou l'autre créent les paysages multiples de cette nouvelle étape de la mondialisation. En Éthiopie, le principal bailleur et opérateur de cette mise aux normes sanitaires animales est l'USAID, qui a investi de manière croissante (dans l'absolu et relativement à ses aides globales à l'Éthiopie) dans le secteur animal.

Au sein de la ligne «Trade capacity building Assistance» de l'aide américaine en Éthiopie, la part destinée au développement pastoral et à l'accès du bétail aux marchés connaît une envolée entre 2002 et $2011^{6}$. Ainsi, cette part est négligeable en 2002, soit 18243 \$ sur une aide totale d'un demi-million de \$. Elle passe à $10 \%$ en 2003 avec la mise en place, en partenariat avec la Livestock Marketing Authority éthiopienne, de deux programmes. Le premier, PARIMA (Pastoral Risk Management), est initialement destiné à l'amélioration des conditions de vie des éleveurs de la région Borana en Oromia. On verra plus bas comment ce programme fait système avec l'arrivée cooptée par l'État d'investisseurs arabes ayant échoué en région somali (ERSE). Le second, LMIS (Livestock Marketing Information System), crée un réseau de relevée des prix sur une cinquantaine de marchés dans tout le pays. En 2005, sur 21 millions de dollars d'accompagnement au renforcement des échanges, $20 \%$ sont destinés aux projets visant directement le bétail, notamment avec l'introduction du programme Pastoral Livelihood Initiative (PLI), qui englobe désormais le LMIS. Le reste est consacré à la mise aux normes en vue d'une entrée à l'OMC. En 2006, le PLI et un nouveau programme, Sanitary and Phytosanitary Standard and Livestock and Meat Marketing Program (SPS/LMM) totalisent près de $50 \%$ de l'assistance financière au commerce. En 2011, c'est 90 \% de cette aide qui est consacrée au commerce de bétail, uniquement via le programme SPS/LMM. Ainsi, non seulement l'Éthiopie réussit à capter des aides importantes sur ce secteur, mais cela semble également intéresser les États-Unis d'investir dans les régions pastorales, en 
accompagnement de leur implantation militaire dans la région (à Djibouti et en Éthiopie).

Cette nouvelle normativité sanitaire mondiale permet à l'Éthiopie de réinvestir le secteur animal en sautant les étapes, l'État étant le seul acteur habilité à certifier la santé des animaux exportés. En ERSE, cette aide américaine va principalement se traduire par la construction d'infrastructures telles que des marchés, des programmes de compensation des sécheresses. Créant ainsi les bases d'une structure parallèle inutile à la vieille garde négociante somali, ces actions visent à faciliter l'introduction de nouveaux acteurs dans la filière.

Les aides internationales et la signature d'accords bilatéraux avec Djibouti et le Soudan pour les échanges transfrontaliers et avec l'Égypte pour le commerce de bétail offrent à l'Éthiopie son entrée dans ce secteur économique. Ces mesures permettent l'intégration du commerce transfrontalier de bétail sur les frontières éthio-soudanaise (Metema et Humera) et éthio-djiboutienne (dans une moindre mesure) et un début de maillage du territoire avec des infrastructures d'exportation (construction de marchés aux bestiaux destinés à l'exportation, planification de la construction de quarantaines animales et de check-points sanitaires aux frontières orientales - même si ces derniers ne sont pas effectifs-. L'accord avec l'Égypte permet à l'Éthiopie (ici indissociable du parti et de l'État) de coopter des négociants des hautes terres vers les nouveaux marchés. Les statistiques des exportations légales en sont améliorées : la part du bétail vivant dans les exportations éthiopiennes est multipliée par 6 entre 2003 et 2006 et le bétail vivant passe $\mathrm{du} 19^{\mathrm{e}}$ au $7^{\mathrm{e}}$ poste des exportations.

Ce volontarisme ne sera pas suffisant pourtant pour "récupérer» le bétail et les marchés les plus lucratifs des mains des grands commerçants somali. Si c'était le cas, une expertise réalisée pour l'IGAD affirme que les seules exportations de bétail vivant participeraient à hauteur de $17 \%$ des revenus des exportations contre 3,6\% aujourd'hui (Behnke, 2011).

21 Les voies du désenclavement économique éthiopien, à l'image de la politique menée dans les régions somali intérieure ou limitrophes sont guidées par la contradiction permanente entre géopolitique intérieure et nécessité économique nationale. À cet égard, les récentes évolutions du secteur animal révèlent la force de la frontière cognitive entre hautes-terres et basses-terres orientales de l'Éthiopie.

\section{Le bétail mondialisé et le renforcement de la frontière cognitive entre les hautes-terres et les basses-terres orientales}

Alors que la filière d'exportation de bétail vivant est dominée par les producteurs et les négociants somali transfrontaliers qui structurent l'espace oriental de la Corne de l'Afrique et de l'Éthiopie depuis plusieurs centaines d'années et alors qu'il existe une frontière culturelle forte divisant l'Éthiopie entre les hautes-terres et les basses-terres pastorales, quel peut être l'impact de la mise aux normes de ce secteur économique sur l'organisation pré-existante? 


\section{Un système somali dans un système Corne.} ou non situées dans le territoire éthiopien, «Éthiopiens» ${ }^{7}$ et Somali sont dépendants les uns des autres: sans l'Éthiopie, pas de commerce de transit pour les Somali dont les ports seraient sous-utilisés ; sans la coopération des populations côtières et des marges pastorales, l'Éthiopie n'a pas d'accès à la mer. Cette complémentarité géographique, immuable, semble exacerber les différences entre les populations des hautes-terres et celles des basses-terres connectées au littoral de la Corne de l'Afrique. Plus récemment, le développement des corridors de désenclavement en forme de tunnels accroît l'éloignement des régions périphériques qu'ils traversent. $S$ 'ils accélèrent le transit des produits arrivés légalement, ils favorisent également l'informalité des acteurs extérieurs au corridor.

betail est la seule ressource qui permet aux Somali quel que soit leur État (aujourd'hui Éthiopie, Somalie, Somaliland, Djibouti, Kenya) de former un système géoéconomique indépendant des hautes terres éthiopiennes, seul à permettre de s'affranchir d'une dépendance politique. Ils ne le vendent pas à «l'Éthiopie», ils ne l'achètent pas à «l'Éthiopie», ils n'ont pas besoin de «l'Éthiopie» pour le faire transiter dans la mesure où le bétail vivant est exporté par voie maritime à partir des ports somali de Berbera, Djibouti ou Bosaaso, ou encore traversent les espaces somali éthiopiens et kenyans avant d'arriver sur les marchés kenyans. Il y a donc deux manières de présenter l'espace du commerce de bétail : celle des grands négociants somali qui le perçoivent dans sa dimension ethnique et transfrontalière selon une carte mentale où la frontière étatique est une discontinuité spatiale mineure; l'autre approche cantonne l'analyse à des territoires nationaux administratifs. On ne peut interpréter la situation relative du bétail de l'ERSE en Éthiopie sans prendre en compte sa situation relative dans l'espace somali.

Ainsi, l'ombre des "autres Somalies " plane sur l'étude d'une ERSE territorialement circonscrite à l'Éthiopie. Cette vision superposée de deux systèmes de représentation permet d'appréhender à la fois l'interconnexion des échelles et la bataille de ces deux mondes pour être arrimés aux marchés arabes, échelle supérieure des échanges.

Par ailleurs, le bétail est la propriété des familles et des clans somali. Mobile, il échappe facilement au contrôle, aux statistiques et à l'impôt et s'insère dans un système de pensée étranger à celui des hautes-terres comme en témoigne le recensement du bétail dans l'ERSE, où ne sont recensés que les animaux des ménages sédentaires, dans la poignée de woreda agro-pastoraux que compte l'État régional somali d'Éthiopie (ERSE).

Les pratiques des exportateurs formels et informels offrent une traduction spatiale à ces propos. Les exportateurs cooptés par l'Éthiopie sont les principaux utilisateurs des nouvelles infrastructures: quarantaine de Dire Dawa, ports secs de chargement du bétail sur des camions, abattoirs d'exportation et routes, points nodaux et lignes qui vont dessiner sur le territoire éthiopien la frontière entre les deux systèmes. Cette ligne cantonne de fait le bétail et les commerçants de l'ERSE dans un système d'échange trans-somali, plus simple et surtout plus court. En effet, la carte indique que les points de chargement du bétail sont tous situés sur les contours extérieurs de l'ERSE, le long des routes développées en priorité, afin d'en capter le bétail sans retombées économiques directes pour la région. Ce sont deux systèmes parallèles qui se confrontent, l'un anciennement mondialisé dans le sens où il est confronté depuis

EchoGéo, 31 | 2015 
longtemps aux concurrents internationaux et a montré sa résilience, mais qui, côté éthiopien, fonctionne en utilisant a minima des infrastructures qui ne lui sont pas destinées. L'autre système bénéficie de la mise aux normes du secteur, enfant d'une autre forme de mondialisation, normative, cette fois. Ses acteurs sont intégrés au triptyque décrit plus haut mais n'ont pas l'ancienneté des réseaux, notamment d'approvisionnement.

Illustration 1 - Exportations officielles de bétail en Éthiopie : une frontière intérieure matérialisée par les infrastructures

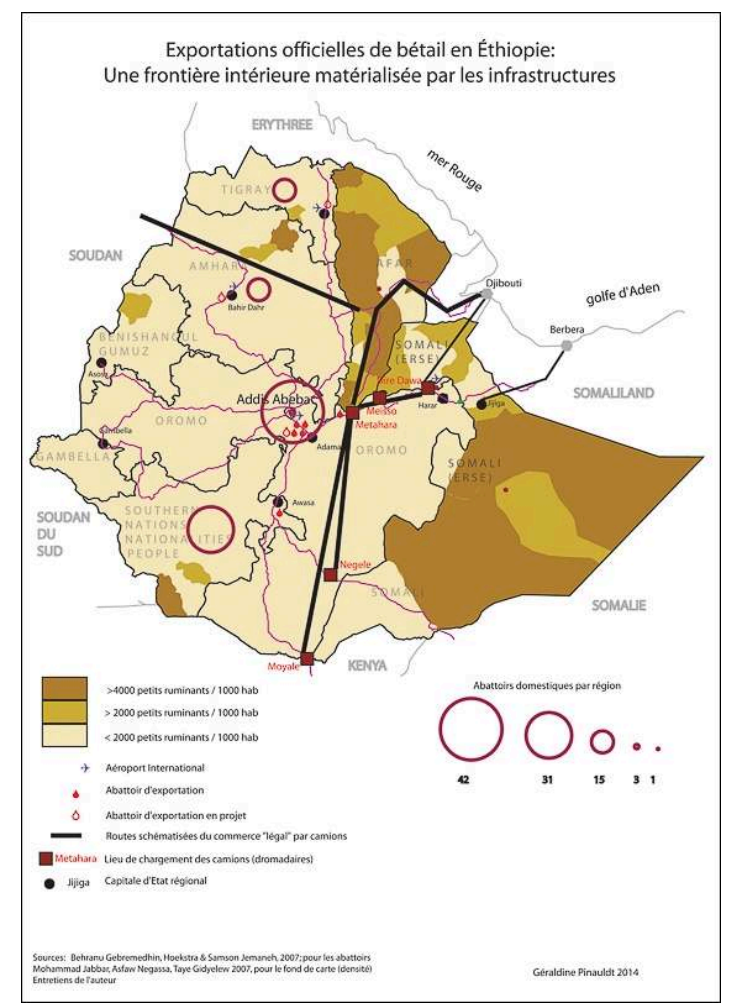

Sources : Behranu Gebeernedlhin, Hoekstra et Samson Jermaneh, 2007 pour les abattoirs ;

Mohammad Jabbar, Asfaw Negassa \& Taye Gidyelew, 2007, pour le fond de carte (densité) ; entretiens de l'auteur. G. Pinauldt, 2014.

\section{Le "propriétaire de l'Éthiopie" et le bétail des Somali}

L'ensemble de ce paragraphe est une reconstitution effectuée par l'auteur entre 2008 et 2010 à partir de différentes sources, principalement orales (grands commerçants de bétail, vétérinaires d'État éthiopiens, intermédiaires). D'autres sources écrites citées plus loin complètent l'analyse.

L'exemple de la filière bétail Elfora du groupe MIDROC du milliardaire éthio-saoudien Mohamed $\mathrm{Al}$ Amoudi, révèle la difficulté à créer Un réseau d'approvisionnement alors même que la ressource est abondante, que les infrastructures existent, que le marché d'exportation existe. Dans le cadre du commerce de bétail, le pouvoir n'est plus du même côté de la frontière cognitive.

Intérêts publics et intérêts privés s'enchevêtrent sans qu'il soit toujours aisé de les distinguer. Difficile, d'abord, de séparer les stratégies économiques ou patriotiques du milliardaire, qui participe personnellement à la levée de l'embargo pour l'Éthiopie, de 
celles de sa filière Elfora, qui devient le principal bénéficiaire de la levée de l'embargo. Difficile ensuite de dissocier les objectifs de l'État éthiopien qui a pour double dessein en cherchant à capter les filières somali d'augmenter ses exportations de viande tout en accédant à un territoire de production qui lui échappe, tant sur des bases économiques que sécuritaires. Enfin, le groupe MIDROC est le plus grand groupe privé éthiopien et $\mathrm{Al}$ Amoudi lui-même est connu pour entretenir des relations privilégiées avec le pouvoir éthiopien.

31 L'Éthiopie entre relativement tard sur le marché international de bétail, et bénéficie pour cela de l'entremise de Mohamed Al Amoudi qui a connaissance à la fois des bénéfices réalisables sur le marché saoudien, de l'importance du cheptel éthiopien et de sa très faible valorisation par l'exportation.

32 La difficulté consiste à capter le bétail dans les régions de production (notamment les régions ayant connaissance de la "qualité export"). Or de ce point de vue, le bétail le plus lucratif du territoire est élevé en région somali, dont, on l'a vu, les réseaux sont anciens et fonctionnels. Les plus grands négociants de bétail de la région sont Somalilandais, parfois binationaux ou utilisant des réseaux d'intermédiaires binationaux ou locaux.

33 En 1997, dans le cadre de la politique éthiopienne de privatisation, le groupe MIDROC d'Al Amoudi rachète les sept entreprises nationales de transformation et d'exportation de bétail qui font alors partie de l'Ethiopian Meat Agency. Les exportations éthiopiennes sont cependant bloquées l'année suivante par les embargos sanitaires de 1998 et 2000 qui touchent l'ensemble des pays de la Corne de l'Afrique.

Le personnage d'Al Amoudi s'investit personnellement dans la négociation saoudoéthiopienne. En 2002, l'embargo est levé pour les exportations éthiopiennes de bétail et de viande. Le magnat binational y est vraisemblablement associé : il fait partie, aux côtés d'un autre investisseur saoudien, d'une délégation saoudienne qui visite les infrastructures éthiopiennes de transformation du bétail, d'exportation de viande et accorde une levée de l'embargo pour l'Éthiopie. La délégation saoudienne promet d'investir largement dans le secteur du bétail, via une coopération vétérinaire et l'investissement dans des infrastructures d'exportation.

35 La coopération saoudienne et la levée de l'embargo donne une belle occasion à l'État éthiopien d'établir une concurrence avec les commerçants Somalilandais "nés en Éthiopie"8. Cette entrée sur le marché saoudien pourrait permettre d'inverser enfin le flux du bétail de la région somali, dont le mouton à tête noire produit une viande particulièrement appréciée en Arabie saoudite. Dans cet élan, l'État tente des incitations à l'installation de commerçants originaires d'autres régions éthiopiennes en ERSE. Cette compétition doit permettre des exportations légales (donc taxées) du bétail et conjointement l'approvisionnement des usines de viande rachetées par la filiale agroalimentaire du groupe MIDROC, Elfora. Le gouvernement fédéral, avec l'appui d'une partie du gouvernement régional somali, y voit aussi une occasion d'éliminer totalement l'usage du Shilling somali (et somalilandais), alors encore majoritairement utilisé dans l'ERSE, au profit du Birr éthiopien et ainsi franchir un pas dans l'intégration nationale de la région. Cette annonce suscite de vifs débats dans la région somali'. Finalement, l'incitation à l'installation de commerçants non-somali se révèle moins forte que la structuration des réseaux existants et seuls quelques acheteurs "éthiopiens-non-somali" commencent à pénétrer cet espace, en restant toutefois contenus dans les marchés-lieux (construits en durs dans le cadre d'un programme de 
maillage financé par l'USAID). L'ouverture du marché égyptien pour l'Éthiopie est une occasion d'introduire de nouveaux acteurs. En effet les réseaux somali y sont moins solides que pour la péninsule arabique et c'est au départ par l'intermédiaire de l'État éthiopien que les négociants vont entrer en contact avec les importateurs égyptiens ${ }^{10}$.

Parallèlement, la même année, Al Amoudi participe, au sein d'une délégation éthiopienne, à des négociations avec le Somaliland ${ }^{11}$ en vue d'un contrat permettant l'exportation du bétail "éthiopien" par le port de Berbera, en échange de la promesse de lever l'embargo pour le Somaliland. Les négociations n'aboutiront pas : le risque est trop grand pour le Somaliland, avec une levée de l'embargo conditionnée par l'Ethiopie, de perdre ses revenus vitaux tirés de l'exportation de bétail - même en période d'embargo ${ }^{12}$-. De plus les exportateurs somalilandais risquent de se voir coupés du marché saoudien au profit du groupe d'Al Amoudi et de grands commerçants éthiopiens, dont ils soupçonnent qu'ils pourraient être favorisés. Pour les Somalilandais, et finalement les Somali d'Éthiopie ${ }^{13}$, ce projet reviendrait à faire acheter le bétail somali par l'Éthiopie pour le revendre à l'Arabie saoudite via le Somaliland.

Ne parvenant finalement à accéder ni au port de Berbera, ni en suffisance au bétail de la région somali d'Éthiopie, l'Éthiopie et l'entreprise Elfora, dont les objectifs convergent, se détournent des exportations de bétail vivant pour se concentrer sur les complexes de viande (abattoirs et usines de conditionnement). En effet, l'Éthiopie étant enclavée, les exportateurs éthiopiens ne peuvent pas exporter de bétail vivant vers la péninsule arabique dans la mesure où les ports d'exportation se situent dans des États encore sous embargo.

Tous les abattoirs d'exportation se situent à l'ouest de la frontière culturelle entre les mondes "somali" et "éthiopien" et loin des filières du fameux mouton à tête noire. Deux choix sont possibles : soit descendre ses usines vers les basses terres de l'est (au risque de ne pas réussir à pénétrer les filières), soit "se contenter" des vaches des hautesterres, d'un moindre potentiel pour l'exportation vivante, alors que vraisemblablement le bétail somali était l'atout premier de filiale animale. Elfora possède bien une filiale à Dire Dawa, à l'entrée de l'ERSE mais peine autant que les autres abattoirs à trouver les animaux nécessaires à son fonctionnement.

En 2004, le groupe MIDROC crée TransNation Airways, une compagnie aérienne de transport de fret et accessoirement de passagers, dont l'objectif premier est le transport de la viande produite par Elfora. (Addis Tribune, 23 juin 2004). Le groupe entier est engagé dans la structuration du secteur d'exportation de produits animaux. Afin d'accéder au bétail de qualité export, Elfora collabore finalement au programme de développement PARIMA (Pastoral Risk Management on East Africa's Rangeland), financé par l'USAID, destiné à intégrer le bétail des éleveurs Borana en Oromiya au marché d'exportation. En effet, à partir de 2003, ce programme, qui existe depuis 1997, est membre d'un comité dirigé par l'association éthiopienne des exportateurs de bétail et de viande et dont le secrétariat est assuré par le Livestock and Fisheries Marketing Department du ministère éthiopien de l'Agriculture. Une évaluation mise en place par le Comité et PARIMA montre que l'ensemble des abattoirs d'exportation fonctionnent en sous-capacité. La région Borana (au sud du pays, en Oromiya), au cœur du projet PARIMA, et ne bénéficiant vraisemblablement pas des réseaux anciens des pasteurs somali pour accéder au marché international devient le centre d'un système liant l'État, 
les ONG et les grands industriels de la viande, dont le leader est Elfora (Solomon Desta, 2006).

Ce programme devient la principale source d'approvisionnement de son abattoir de Debre Zeit, montrant ainsi la convergence des "nouveaux acteurs " d'une "mise en mondialisation sectorielle" de l'Éthiopie et entérinant le fonctionnement parallèle de deux marchés animaux.

41 Selon les acteurs considérés, la réussite du projet est à relativiser. En effet, à l'échelle de l'Éthiopie, les exportations de viande ont été multipliées depuis sa mise en place et leur part dans les exportations est de plus en plus importante. En revanche, pour les industriels, dont le leader est sans conteste Elfora, le succès est plus relatif: les abattoirs d'exportation fonctionnent toujours en sous-capacité et le programme n'a pas suffi à construire des relations de confiances entre les éleveurs et les courtiers nommés par les industriels.

42 Cette coopération entre des acteurs de nature et d'échelle d'intervention différentes obtient un succès relatif, mais permet néanmoins l'entrée de l'Éthiopie sur le marché international du bétail. Cependant, l'un des objectifs de départ, à savoir les réseaux orientaux du commerce, n'est pas atteint, et on assiste, après une forme d'engouement pour ces espaces et leurs ressources, à une relégation dans l'oubli (dans l'inconscient national?). Aussi est-il intéressant de constater que l'articulation d'un secteur de l'économie au commerce mondial et mondialisé va, dans ce cas précis, renforcer un déséquilibre. Bien que les statistiques du groupe soient difficiles d'accès, la nonexistence d'Elfora dans le discours des grands négociants de bétail en ERSE souligne tout de même un échec conséquent au regard de la puissance économique du groupe et de la taille du cheptel de la région somali.

43 L'action conjuguée des acteurs de la mise en mondialisation par les normes (programmes de l'USAID, organisations internationales ou ONG qui en sont les opérateurs), d'un grand acteur économique et de l'État éthiopien contribue à renforcer les différentiels de développement et de perception de part et d'autre de la frontière cognitive à l'échelle du territoire éthiopien.

\section{Conclusion}

Entre 1998 et le début des années 2010, le global formalisé accède à l'ERSE via Addis Abeba, sans réelle émancipation d'une administration locale. Les acteurs des projets connectés au monde normalisé sont des acteurs choisis, dans une double volonté éthiopienne de développement économique et de contrôle politique par le marché. Ces volontés contradictoires vouent ces projets à un développement limité alors que les acteurs sectoriels traditionnels sont déjà organisés, déjà mondialisés et que la certification se traite dans les villes portuaires. Aussi, la question d'un stock grabbing coopté par Addis-Abeba hors de contrôle des acteurs locaux n'est pas encore posée pour le bétail de la région somali d'Éthiopie. Toutefois, les territoires somali même organisés par les réseaux informels pré-existants, s'ils s'affranchissent d'Addis Abeba, restent dans une relation de dépendance aux importations saoudiennes. Cette asymétrie a été perturbée par l'entrée de l'Arabie Saoudite dans une mondialisation normative contraignante. 
Depuis 2010, une nouvelle administration régionale offrant en gage à Addis Abeba la sécurisation de l'ERSE a pu négocier une autonomisation régionale, conformément au statut des États régionaux dans la Constitution. La décentralisation se fait plus réelle et touche depuis récemment des pans important de l'économie locale dont les portées socio-spatiales sont à venir. Avec une administration perçue comme affranchie du pouvoir fédéral, les acteurs locaux s'autorisent, encore timidement, à investir dans leur région. Dans le cas du bétail, un premier projet d'abattoir d'exportation porté par un entrepreneur somali de la diaspora est en cours de réalisation à proximité de Jijiga, là où de grands projets d'industrie intégrée de ranch et de transformation du bétail sont annoncés par Addis-Abeba depuis dix ans mais n'ont jamais pu être réalisés. Alors que les précédents projets étaient jusqu'alors imposés à l'État régional depuis Addis Abeba, on assiste, depuis une période très contemporaine à un embryon d'inversement de la hiérarchie scalaire avec des propositions venues de la région acceptées par l'administration fédérale, la région jouant un nouveau rôle d'intermédiation entre les porteurs de projets (déjà mondialisés, venant de la diaspora et/ou ayant des réseaux importants dans les pays du golfe) et l'État fédéral. Ces derniers développements sont trop neufs pour que des conséquences spatiales puissent en être tirées à long terme, mais ils indiquent une direction nouvelle.

\section{BIBLIOGRAPHIE}

Addis Tribune, 23 juin 2004. Midroc establishes Airline Company here.

Barnes C., 2010. The Ethiopian-British Somaliland Boundary. In Dereje Feyissa and Hoehne M. V. (Eds.), Border and Borderlands as Resources in the Horn of Africa, Suffolk, James Currey, p 122-131.

Behnke R., 2011. The Contribution of livestock sector to the Ethiopian economy. Part II, IGAD LPI $\mathrm{n}^{\circ} 02-2011,43 \mathrm{p}$.

Faye B., 1990. Éleveurs d'Éthiopie. Paris, Karthala, 194 p.

Federal Democratic Republic of Ethiopia, Central Agricultural Census Commission, 2003. Ethiopian Agricultural Sample Enumeration, 2001/02 (1994 E.C.). Results for Somali Region. Statistical Report on socio economic characteristics of the population in agricultural households, land use, area and production of crops, farm management practices, Livestock and Farm implements. Addis-Abeba.

Hagmann T., 2005. Challenges of Decentralisation in Ethiopia's Somali Region. Review of African Political Economy, 32, p. 449-455.

Jabbar M., Negassa Asfaw \& Taye Gidyelew, 2007. Geographic Distribution of Cattle and Shoats Populations and their Market suplly Sheds in Ethiopia. Discussion Paper No.2. Improving Market Opportunities, ILRI, Nairobi, Kenya, 57 p.

Pinauldt G., 2009. Épizooties et géographie du commerce du bétail dans la Corne d'Afrique. EchoGéo [En ligne], 8 | 2009, mis en ligne le 30 mars 2009. URL : http://echogeo.revues.org/11021 ; DOI : $10.4000 /$ echogeo. 11021 
Pinauldt G., 2014. L'or vivant des Somali. Des frontières, des troupeaux et des hommes face à la mondialisation des normes : un regard géopolitique sur les exportations de bétail de la Corne de l'Afrique. Thèse de doctorat non publiée, Saint-Denis, Université Paris 8, 352 p.

Planel, S., 2007. Du centralisme à l'éthnofédéralisme. La décentralisation conservatrice de l'Éthiopie. Afrique contemporaine, 221, p. 87-107.

Solomon Desta, Getachew Gebru \& all, 2006. Linking pastoralists and exporters in a livestock marketing chain: recent experiences from Ethiopia. In McPeak J. et Little P. D., Pastoral Livestock Marketing in Eastern Africa. Warwickshire, Intermediate Technology Publication, Schumacher Centre for Technology and Development, p. 109-127.

\section{NOTES}

1. Entre $25 \%$ et $75 \%$ selon les sources et selon les années.

2. Dès lors, le bétail de la Corne de l'Afrique a été utilisé pour l'approvisionnement en viande des garnisons britanniques établies à Aden.

3. Si les États importateurs considèrent, suivant les recommandations de l'OIE, que pour accéder aux ports d'exportation le bétail séjourne trop longtemps dans une zone endémique, le certificat n'est pas valide. De plus, entre 2006 et 2009, la quarantaine animale de Djibouti était la seule à avoir obtenu la reconnaissance du Ministère saoudien de l'Agriculture. À cette période, l'Égypte est le seul État qui reconnaît les certificats issus par la quarantaine animale de Dire-Dawa dans le cadre d'un accord bilatéral encadrant les échanges entre les deux États. Voir pour cela Pinauldt, 2009.

4. Voir Planel, 2007.

5. Le discours public éthiopien sur l'ERSE oscille entre la virginité supposée des terres somali, considérées comme un front pionnier pour l'agriculture et la menace intérieure que représente sa population, les terres somali font alors figure de front guerrier. C'est d'ailleurs cette dualité du discours qui va longtemps freiner l'engagement éthiopien dans le développement de cette région, sans pour autant souhaiter son détachement de l'Éthiopie.

6. USG Trade Capacity Building Database, http://qesdb.cdie.org/tcb/index.html, USAID Development Information Services, consulté le 5 juillet 2013.

7. Lorsqu'ils sont utilisés entre guillemets, le terme «Éthiopie» et ses déclinaisons font référence non à l'État éthiopien ou à la nationalité éthiopienne, mais à la représentation répandue (et notamment dans une vision somali) qu'est éthiopien un éthiopien des hautes-terres (et dont on sous-entend qu'il est chrétien, agriculteur et sédentaire), par opposition aux peuples périphériques, notamment pastoraux, et particulièrement somali : musulman, éleveur et nomade. Si cette vision semble pour le moins essentialiste, elle correspond néanmoins à des représentations opérationnelles sur le terrain.

8. Ré-utilisant ainsi une rhétorique employée aux cours de la première moitié du XXe siècle dans les négociations avec le protectorat britannique du Somaliland, qui consistait à qualifier de sujets britanniques les Somali issus du clan majoritaire au Somaliland (mais dont les territoires s'étendent au-delà de la frontière, en Éthiopie). Cette rhétorique rappelle néanmoins la difficulté pour l'Éthiopie à distinguer parmi cette population nomade et transfrontalière qui est Éthiopien et qui ne l'est pas. Les mêmes questions se posent d'ailleurs quant à la nationalité du bétail.

9. Haatuf, $\mathrm{n}^{\circ} 120,22$ juillet 2002. "Etoobiya oo bilaabaysa dhoofinta xoolaha nool" (L'Éthiopie commence l'exportation du bétail vivant) http://www.haatuf.net/ Archive2002/00012000.htm, consulté le 15 mars 2013. Haatuf est un quotidien de la presse papier du Somaliland. Il s'agit du journal qui possède les plus anciennes archives 
en ligne de ses articles. Les actualités de l'État régional somali d'Éthiopie sont d'une manière générale très peu relatées dans les media éthiopiens.

10. Entretiens réalisés en 2009 et 2010 avec plusieurs négociants non-somali de bétail achetant du bétail originaire d'ERSE.

11. Entretiens réalisés entre 2008 et 2009 avec l'un des membres de la délégation éthiopienne au Somaliland.

12. Le bétail continue d'être exporté depuis Berbera - de manière moins lucrative - vers le Yémen qui a levé l'embargo sur le Somaliland en 2002, et les Émirats arabes unis.

13. Le double échec de l'introduction de commerçants non-somali en ERSE et des négociations avec le Somaliland fait apparaître que malgré une lourde histoire de conflits intra-Somali opposant particulièrement des clans présents en ERSE et au Somaliland, la solidarité somali prime lorsqu'il s'agit de bétail dans un rapport aux "Ethiopiens" (pouvoir éthiopien).

\section{RÉSUMÉS}

Le commerce de bétail en Éthiopie est marqué par une frontière intérieure que dessine la vallée de l'Awash/vallée du Rift et qui matérialise une frontière cognitive entre les basses-terres Somali et les hautes-terres de l'Éthiopie. À l'ouest de cette ligne, le bétail nourrit principalement la consommation éthiopienne et à l'est, largement informel et dominé par les grands commerçants somali, il est exporté vers la péninsule arabique via le Somaliland. Profitant d'une conjonction de facteurs accélérant la "mise en mondialisation" du commerce de bétail, l'État éthiopien conjugue la captation des aides internationales pour des programmes d'accès aux marchés et de mise aux normes commerciales et vétérinaires avec une ouverture nouvelle à des investisseurs saoudiens. Mais ces projets se heurtent à la résistance d'un système géoéconomique pluri-centenaire dans une région Somali d'Éthiopie pas encore tout à fait acquise à la cause éthiopienne, pour lequel le bétail demeure une richesse non aliénable par l'État. Ici, la mise en mondialisation de l'Éthiopie, sous la forme d'une normalisation des échanges internationaux, donne à une occasion l'État éthiopien de mieux maîtriser un territoire périphérique et sa ressource. Cette utilisation des outils normatifs permet-elle d'intégrer l'économie de la région Somali d'Éthiopie et ainsi de rééquilibrer le territoire national, ou ce processus renforce-t-il cette frontière culturelle intérieure?

\section{INDEX}

Mots-clés : commerce de bétail, frontière cognitive, mondialisation des normes, Arabie Saoudite, Somali, État régional Somali d'Éthiopie

\section{AUTEUR \\ GÉRALDINE PINAULDT}

Géraldine Pinauldt, geraldinepinauldt@hotmail.com, est Docteur en Géographie. 